\title{
Temperature and Pressure Effects on the Bending Modulus of Monolayers in a Ternary Microemulsion
}

\author{
Y. Kawabata, ${ }^{1,2, *}$ M. Nagao, ${ }^{3}$ H. Seto, ${ }^{4}$ S. Komura, ${ }^{1}$ T. Takeda, ${ }^{5}$ D. Schwahn, ${ }^{6}$ N. L. Yamada, ${ }^{2}$ and H. Nobutou ${ }^{2}$ \\ ${ }^{1}$ Department of Chemistry, Faculty of Science, Tokyo Metropolitan University, Tokyo 192-0397, Japan \\ ${ }^{2}$ Graduate School of Bio-Sphere Science, Hiroshima University, Higashi-Hiroshima 739-8521, Japan \\ ${ }^{3}$ Institute for Solid State Physics, The University of Tokyo, Tokai 319-1106, Japan \\ ${ }^{4}$ Graduate School of Science, Kyoto University, Kyoto 606-8502, Japan \\ ${ }^{5}$ Faculty of Integrated Arts and Sciences, Hiroshima University, Higashi-Hiroshima 739-8521, Japan \\ ${ }^{6}$ Institut für Festkörperforschung des Forschungszentrums Jülich GmbH, D-52425 Jülich, Germany
}

(Received 21 April 2003; published 6 February 2004)

\begin{abstract}
We performed small-angle neutron scattering and neutron spin echo experiments on a ternary microemulsion composed of ionic surfactant AOT, water, and decane. Thermal fluctuations of monolayers have been investigated as a function of temperature and pressure. The amphiphilic monolayers become more flexible with increasing temperature and more rigid with increasing pressure. These results are consistent with the microscopic picture that the head-head repulsion of the AOT molecules is enhanced at high temperature while an attractive interaction between the hydrophobic tails of the AOT molecules increases at high pressure.
\end{abstract}

DOI: 10.1103/PhysRevLett.92.056103

PACS numbers: 68.05.Gh, 61.12.Ex, 62.50.+p

Electronic, magnetic, or structural properties of condensed matters react to variations in temperature and pressure. In order to investigate the physical properties of condensed matters, the temperature and pressure dependencies of structures and physical quantities are important. In many materials such as simple liquids, gas, or crystalline solids, an increase of temperature has the same effect as a decrease of pressure because the Clausius-Clapeyron relation $d T / d P>0$ is usual.

For a soft condensed matter, however, there are systems such as surfactant solutions in which increasing pressure and decreasing temperature have different effects $(d T / d P<0)$. In a mixture composed of water, decane, and AOT (dioctyl sulfosuccinate sodium salt), a water-inoil droplet phase is formed over a wide range of composition at ambient temperature and pressure [1]. By raising temperature, a single droplet phase undergoes a phase separation [2,3]. A similar phenomenon is observed when pressure is increased $[4,5]$. Hence increasing temperature and increasing pressure play a similar role in the phase behavior of this system at least on a phase behavior. However, such pressure effects on the structural formation [6] have not been investigated in much detail. Especially, only very few studies exist on dynamical properties of microemulsion systems under the variation of pressure and temperature [7].

In this Letter, we present the results of small-angle neutron scattering (SANS) and neutron spin echo (NSE) experiments in the dilute droplet regime of the ternary microemulsion composed of AOT, water, and decane. We discuss the temperature and pressure dependencies of the thermal fluctuations of AOT monolayers. Furthermore, we discuss a microscopic picture of the temperature and pressure effects on AOT monolayers based on the bending energy [8],

$$
H=\int\left[\frac{\kappa}{2}\left(\frac{1}{R_{1}}+\frac{1}{R_{2}}-\frac{2}{R_{\mathrm{S}}}\right)^{2}+\bar{\kappa} \frac{1}{R_{1} R_{2}}\right] d S,
$$

where $R_{1}$ and $R_{2}$ are the principal curvature radii, $R_{\mathrm{S}}$ the spontaneous curvature radius, $\kappa$ the bending modulus, $\bar{\kappa}$ the saddle-splay modulus, and $d S$ an area element. There exist a number of studies to obtain $\kappa$ and $\bar{\kappa}$ in a dilute droplet system by means of NSE [9-11]. We improve here the procedure used in the above studies in order to explore the temperature and pressure dependencies of $\kappa$.

We prepared a dilute droplet sample as described below. AOT (purity, 99\%) was purchased from Sigma Chemical and purified by extraction with benzene. Deuterated water $\left(\mathrm{D}_{2} \mathrm{O}\right)$ and deuterated decane $\left(\mathrm{C}_{10} \mathrm{D}_{22}\right)$ were purchased from Isotec Inc. with isotopic purities of 99.9\%-D for $\mathrm{D}_{2} \mathrm{O}$ and $\mathrm{C}_{10} \mathrm{D}_{22}$. These ingredients were mixed at 5.4 vol. $\%$ of AOT, 4.6 vol. $\%$ of $\mathrm{D}_{2} \mathrm{O}$, and 90 vol. $\%$ of $\mathrm{C}_{10} \mathrm{D}_{22}$, so that the molar ratio of $\mathrm{D}_{2} \mathrm{O}$ to AOT was 18.4, and the volume fraction of droplets was $\phi=0.1$. Because the neutron scattering length densities of $\mathrm{D}_{2} \mathrm{O}$ and $\mathrm{C}_{10} \mathrm{D}_{22}$ are almost the same, only the AOT layers can be observed (shell contrast). The SANS experiments were carried out at the SANS-U and KWS1 diffractometers in JRR3M, JAERI, Japan, and FRJ2, FZJülich, Germany, respectively. The NSE experiments were performed at the ISSP-NSE spectrometer in JAERI [12]. The conditions for each experiment are listed in Table I.

Typical SANS intensities $I(q)$ as a function of momentum transfer $q$ are shown in Fig. 1. The critical scattering due to the droplet density fluctuations in the one-phase region is enhanced with increasing temperature as the 
TABLE I. The experimental settings for SANS-U, KWS1, and ISSP-NSE. $\lambda$ is the neutron wavelength for each spectrometer, and $\delta \lambda / \lambda$ is its resolution (FWHM). $T_{\text {range }}$ and $P_{\text {range }}$ are the range of measured temperature and pressure. $\Delta T$ and $\Delta P$ are temperature and pressure variation steps, respectively. $t$ is the range of NSE time. The pressure variation and the temperature variation in the SANS-U and NSE experiments were done at fixed temperature $(T=298 \mathrm{~K})$ and at fixed pressure $(P=0.1 \mathrm{MPa})$, respectively.

\begin{tabular}{lccccc}
\hline \hline & $\lambda[\AA](\delta \lambda / \lambda \%)$ & $q\left[\AA^{-1}\right]$ & $T_{\text {range }}(\Delta T)[\mathrm{K}]$ & $P_{\text {range }}(\Delta P)[\mathrm{MPa}]$ & $t[\mathrm{~ns}]$ \\
\hline SANS-U & $7.0(10)$ & $0.008 \sim 0.3$ & $284 \sim 353(3)$ & $0.1 \sim 66.5(5)$ & $\ldots .1 \sim 30(10)$ \\
KWS1 & $7.0(20)$ & $0.006 \sim 0.14$ & $286 \sim 298(2)$ & 0.1 & $\ldots .1 \sim 60(20)$ \\
ISSP-NSE & $7.14(18)$ & $0.04 \sim 0.13$ & $284 \sim 338(5)$ & $0.15 \sim 15$ \\
\hline \hline
\end{tabular}

phase separation line is approached from below. The scattering intensity is suppressed at higher temperature in the two-phase region. Similar behavior is observed by changing pressure across the phase separation line. From this observation, we estimated the phase separation temperature to be $T_{\mathrm{ps}}=332 \mathrm{~K}$ for $P=0.1 \mathrm{MPa}$ (ambient pressure), and the phase separation pressure to be $P_{\mathrm{ps}}=$ $43 \mathrm{MPa}$ for $T=298 \mathrm{~K}$ (room temperature).

The SANS profiles were analyzed using the procedure by Gradzielski et al. [13], and we obtained the mean radius of the droplets $R_{0}(=\langle R\rangle)$ and the polydispersity index $p \equiv \sqrt{\left\langle R^{2}\right\rangle /\langle R\rangle^{2}-1}$. The polydispersity index is given by

$$
p^{2}=\frac{k_{\mathrm{B}} T}{4 \pi}\left[6(2 \kappa+\bar{\kappa})-8 \kappa \frac{R_{0}}{R_{s}}+\frac{3 k_{\mathrm{B}} T}{2 \pi} f(\phi)\right]^{-1}
$$

where $k_{\mathrm{B}}$ is the Boltzmann constant and $f(\phi)$ the mixing entropy per droplet as a function of the volume fraction $\phi$ [14]. For $T=298 \mathrm{~K}$ and $P=0.1 \mathrm{MPa}$, we obtained $p=$ 0.16 and $R_{0}=32 \AA$ from the SANS data. When temperature is increased to $329 \mathrm{~K}, p=0.15$, and $R_{0}$ decreased to $28 \AA$. On the other hand, $R_{0}$ did not depend on pressure very much.

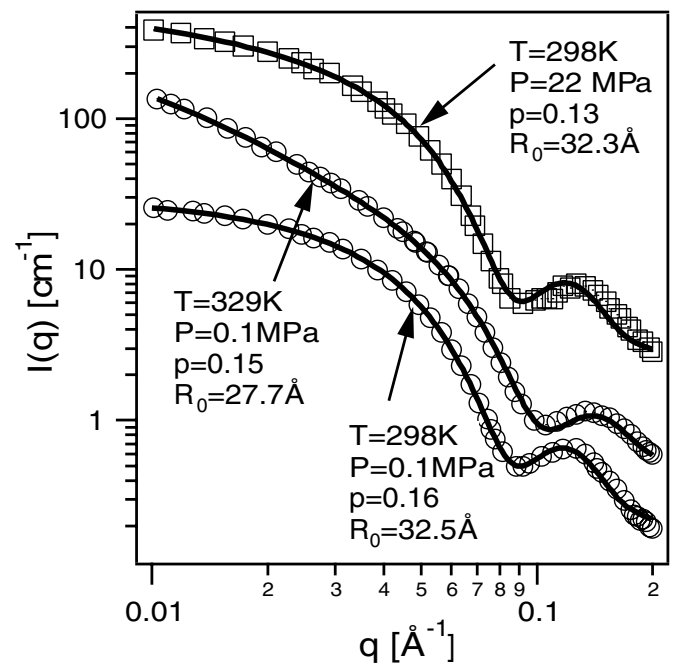

FIG. 1. SANS profiles observed at three different conditions. The data at $T=298 \mathrm{~K}$ (room temperature) and $P=0.1 \mathrm{MPa}$ (ambient pressure) is shown in the absolute unit. Two other curves are multiplied by factors of 2 and 4, respectively.
From the NSE experiment, we obtained the normalized intermediate scattering function $I(q, t) / I(q, 0)$ which we analyzed following the procedure by Huang et al. $[9,10]$. In their treatment, $I(q, t) / I(q, 0)$ is given by

$$
\begin{gathered}
I(q, t) / I(q, 0)=\exp \left[-D_{\text {eff }}(q) q^{2} t\right], \\
D_{\text {eff }}(q)=D_{\mathrm{tr}}+D_{\text {def }}(q),
\end{gathered}
$$

where $D_{\text {eff }}(q)$ is the effective diffusion coefficient, with $D_{\text {tr }}=D_{\text {eff }}(q=0)$ the translational diffusion coefficient of droplets, and $D_{\text {def }}$ the diffusion coefficient in terms of droplet shape deformations. We found that the hydrodynamic radius obtained from $D_{\text {tr }}$ with the Stokes's law was about 2 times larger than the droplet radius $R_{0}$ determined by SANS. The values from the fitting of $D_{\mathrm{tr}}$ at each condition are shown by the arrows in Fig. 2 .

Figure 2 shows $D_{\text {eff }}(q)$ as a function of $q$. It is clearly seen that $D_{\text {eff }}(q)$ becomes larger with increasing temperature and smaller with increasing pressure. Hence the increase of temperature and pressure give rise to opposite effects on the diffusion coefficient of droplets.

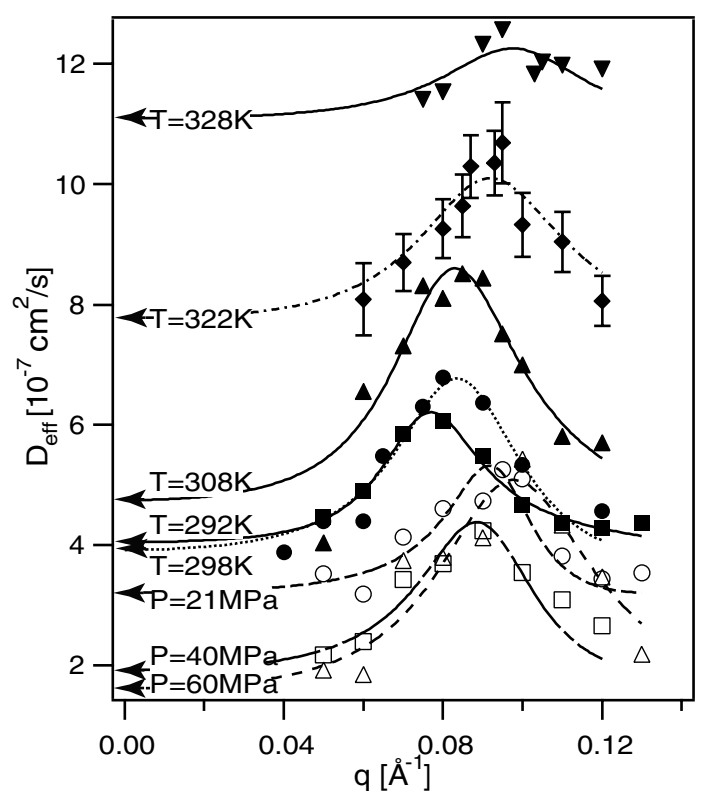

FIG. 2. $D_{\text {eff }}$ obtained from NSE data at various conditions. Lines are fitting results to Eq. (4). The values of $D_{\text {eff }}$ at $q=0$ correspond to $D_{\mathrm{tr}}$, which is shown by the arrows. 
According to Ref. [14], $D_{\text {def }}(q)$ is given by

$$
\begin{gathered}
D_{\mathrm{def}}(q)=\frac{5 \lambda_{2} f_{2}\left(q R_{0}\right)\left\langle\left|a_{2}\right|^{2}\right\rangle}{q^{2}\left[4 \pi\left[j_{0}\left(q R_{0}\right)\right]^{2}+5 f_{2}\left(q R_{0}\right)\left\langle\left|a_{2}\right|^{2}\right\rangle\right]}, \\
f_{2}\left(q R_{0}\right)=\left[4 j_{2}\left(q R_{0}\right)-q R_{0} j_{3}\left(q R_{0}\right)\right]^{2} .
\end{gathered}
$$

Here, $j_{n}(x)$ is the $n$th spherical Bessel function, $\lambda_{2}$ the damping frequency of the droplet deformation, and $\left\langle\left|a_{2}\right|^{2}\right\rangle$ the mean square displacement of the 2nd-mode spherical harmonic $(n=2)$. We fitted the data by Eq. (4) as shown in Fig. 2, and obtained $\lambda_{2}$ and $\left\langle\left|a_{2}\right|^{2}\right\rangle$.

In Fig. 3, we present the temperature and pressure dependencies of $\left\langle\left|a_{2}\right|^{2}\right\rangle$. We find that the shape fluctuations are enhanced for higher temperature while they are suppressed for higher pressure.

We estimated the bending modulus $\kappa$ of the monolayers. For higher pressure, the viscosity of deuterated decane increases more than that of water. Therefore, we must consider the difference between $\eta^{\prime}$ and $\eta$ which are the viscosities inside and outside a droplet. Thus $\lambda_{2}$ is expressed as [15]

$$
\lambda_{2}=\frac{\kappa}{\eta R_{0}^{3}}\left[4 \frac{R_{0}}{R_{s}}-3 \frac{\bar{\kappa}}{\kappa}-\frac{3 k_{B} T}{4 \pi \kappa} f(\phi)\right] \frac{24 \eta}{23 \eta^{\prime}+32 \eta} .
$$

Combining Eqs. (2) and (7), we get the expression of the bending modulus $\kappa$ [16] with the observable parameters, $p^{2}, R_{0}$, and $\lambda_{2}$,

$$
\kappa=\frac{1}{48}\left(\frac{k_{\mathrm{B}} T}{\pi p^{2}}+\lambda_{2} \eta R_{0}^{3} \frac{23 \eta^{\prime}+32 \eta}{3 \eta}\right),
$$

where $\eta$ and $\eta^{\prime}$ are the bulk viscosities of $\mathrm{D}_{2} \mathrm{O}$ and decane, respectively.

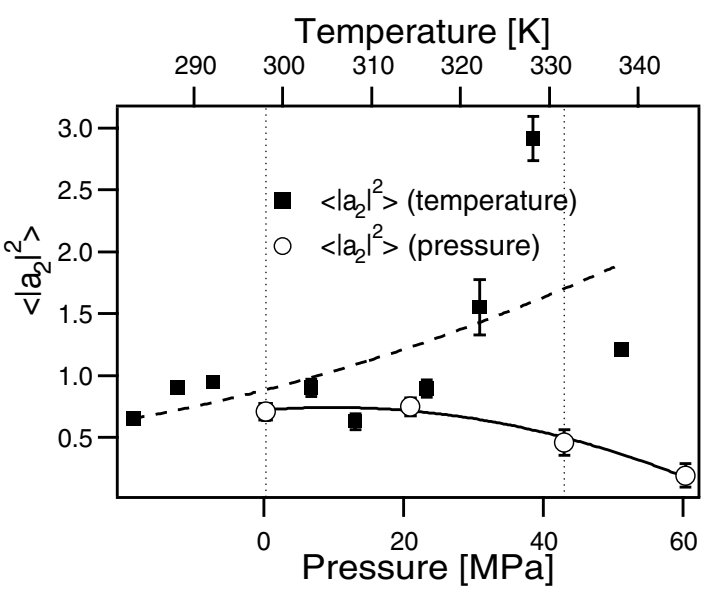

FIG. 3. The temperature and pressure dependencies of $\left\langle\left|a_{2}\right|^{2}\right\rangle$ obtained from Eq. (5). The left dotted line indicates the room temperature and ambient pressure, whereas the right one gives the phase separation temperature and pressure. The shape fluctuation of a droplet becomes larger with increasing temperature, while it becomes smaller with increasing pressure. Curves are drawn to guide the eye.
Figure 4 shows the temperature and pressure dependencies of $\kappa$ obtained from Eq. (8). It is clear that $\kappa$ becomes smaller with increasing temperature while it becomes larger with increasing pressure. For example, $\kappa$ at $T=312 \mathrm{~K}$ was estimated to be $0.26 k_{\mathrm{B}} T$, which was slightly smaller than that at room temperature. On the contrary, $\kappa$ at $P=43 \mathrm{MPa}$ becomes 1.3 times larger than that at ambient pressure $(P=0.1 \mathrm{MPa})$.

From the results of $\left\langle\left|a_{2}\right|^{2}\right\rangle$ and $\kappa$ shown in Figs. 3 and 4, we conclude that the AOT monolayers become more flexible with increasing temperature, while they become more rigid with increasing pressure. These tendencies are consistent with the NSE results obtained by Nagao et al. in the dense droplet regime $(\phi \sim 0.6)$ [18]. Using a model describing single membrane undulations [19], they showed that $\kappa$ at higher temperature was smaller than that at room temperature, and $\kappa$ at higher pressure was larger than that at ambient pressure. Thus, all these results indicate that the temperature effect on the dynamics of the monolayers is opposite to the pressure effect.

The values of $\kappa$ and $\left\langle\left|a_{2}\right|^{2}\right\rangle$ were experimentally obtained as $\kappa \simeq 0.3 k_{\mathrm{B}} T$ and $\left\langle\left|a_{2}\right|^{2}\right\rangle \simeq 0.7$ at ambient temperature and pressure, which are smaller and larger than those in literature [ $\left.\kappa \approx 0.9 k_{\mathrm{B}} T,\left\langle\left|a_{2}\right|^{2}\right\rangle \approx 0.01\right]$ [11]. The origin of the small value of $\kappa$ and the too large value of $\left\langle\left|a_{2}\right|^{2}\right\rangle$ could be the same; the value of $D_{\mathrm{tr}}$ was ambiguous because it was treated as a fit parameter. Hellweg et al. suggested that a combination of dynamic light scattering (DLS) and NSE gives more appropriate value [11]. With our preliminary DLS experiment at ambient temperature and pressure, the values of $\kappa$ and $\left\langle\left|a_{2}\right|^{2}\right\rangle$ were reasonable; $\kappa \approx 0.8 k_{\mathrm{B}} T$ and $\left\langle\left|a_{2}\right|^{2}\right\rangle \approx 0.07$. However, the tendency should be correct from the above results (Figs. 3 and 4)

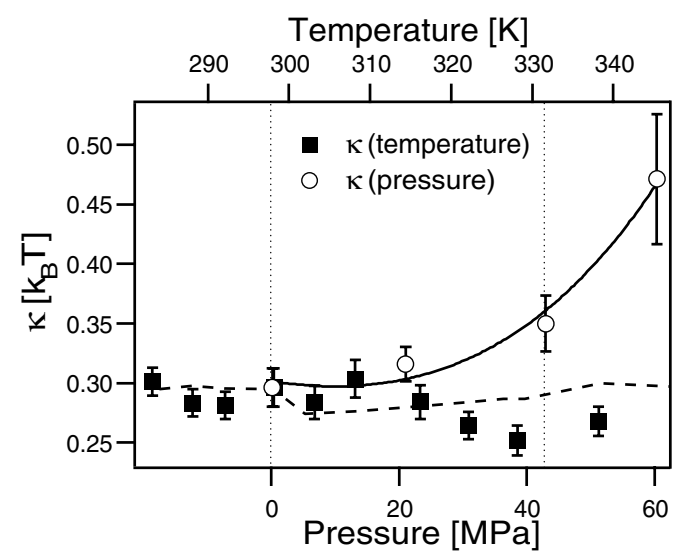

FIG. 4. Temperature and pressure dependencies of $\kappa$ obtained from Eq. (8). The left dotted line indicates the room temperature and ambient pressure, whereas the right one gives the phase separation temperature and pressure. AOT monolayers become flexible with increasing temperature, while they become rigid with increasing pressure. Curves represent the bending modulus $\kappa_{\text {cal }}$ obtained from the calculation according to the model proposed by Würger [17]. The value of $\kappa_{\mathrm{cal}}$ is multiplied by a factor of 0.38 . 


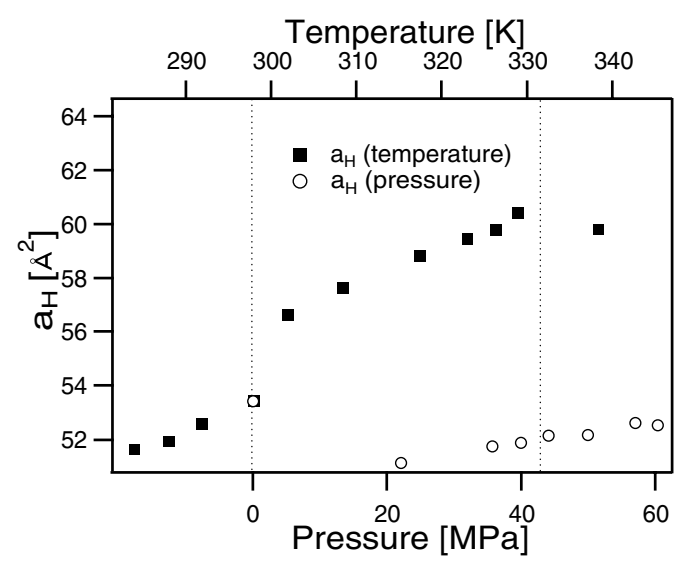

FIG. 5. The temperature and pressure dependencies of area per AOT molecule $a_{\mathrm{H}}$. The left dotted line indicates the room temperature and ambient pressure, whereas the right one gives the phase separation temperature and pressure.

although the absolute values of $\kappa$ and $\left\langle\left|a_{2}\right|^{2}\right\rangle$ might not be very correct.

The microscopic origin of the temperature and pressure dependencies of $\kappa$ has been interpreted as follows $[6,18]$. With increasing temperature, a counterion dissociation from a head group of AOT is enhanced, and an electrostatic head-head repulsive force increases. On the other hand, at high pressure, an attractive interaction between hydrophobic tails of the AOT molecules increases. Thus it is expected that the head area per AOT molecule $a_{\mathrm{H}}$ becomes larger at higher temperature and smaller at higher pressure with the result of the change of monolayer flexibility.

The area per AOT molecule $a_{\mathrm{H}}$ was obtained from the SANS data following the relation, $a_{\mathrm{H}}=4 \pi R_{0}^{2} / n_{\mathrm{s}}$, where $n_{\mathrm{s}}$ is the number of surfactant per droplet. Figure 5 shows the temperature and pressure dependencies of $a_{\mathrm{H}}$. We find that $a_{\mathrm{H}}$ at higher temperature is larger than that at higher pressure. It is clear that the temperature and pressure dependencies of $a_{\mathrm{H}}$ and $\kappa$ are consistent with the microscopic picture described above.

This picture is consistent with a microscopic model describing the bending rigidity [17]. In the model, the interaction between hydrocarbon chains of surfactants is assumed to be a Lennard-Jones potential with the potential minimum $\epsilon$ at an intermolecular distance $\sigma$. As shown by the dashed line in Fig. 4, the temperature dependence of $\kappa$ was reproduced by using this model and the temperature dependence of $a_{\mathrm{H}}$. Note that the absolute value of the calculated $\kappa_{\text {cal }}$ was about $0.8 k_{\mathrm{B}} T$ and the line in the figure was multiplied by the factor of about 0.38 .

The solid line in Fig. 4, representing the pressure dependence of $\kappa$, is a fitting result to the experimental data according to the model. In this fitting procedure, linear pressure dependencies of $\epsilon$ and $\sigma$ were assumed. The fit result reproduced the pressure dependence of $\kappa$, and the potential depth $\epsilon$ increased linearly with $P$, while the potential minimum $\sigma$ was decreased with $P$. This result is also consistent with the microscopic origin of pressure- induced phase transition, in which the attractive interaction between hydrocarbon chains of AOT molecules increases at high pressure. The details of this analysis will be discussed in our following paper.

In summary, we measured the temperature and pressure dependencies of the bending modulus in the ternary microemulsion system for the first time. The result clearly showed that the AOT monolayer becomes more flexible with increasing temperature while more rigid with increasing pressure although the phase transitions induced by temperature and pressure seem to be the same. This evidence was consistent with the microscopic picture of the origins of the phase transitions.

We appreciate Mr. M. Koizumi at Hikari HighPressure Machinery Co. Ltd. for developing the highpressure cell for the NSE experiments. One of the authors (M. N.) was supported by the Grant-in-Aid for Encouragement of Young Scientists (No. 13740234) and (H. S) and (T. T) were also supported by a Grant-in-Aid for Scientific Research (No. 12640377, No. 09640466, No. 13440119) from the Japanese Ministry of Education, Culture, Sports, Science and Technology. Our SANS and NSE experiments at JAERI were done under approval of the Neutron Scattering Program Advisory Committee (Proposal No. 00-199, No. 00200, No. 00-201).

*Electronic address: youheik@comp.metro-u.ac.jp

[1] M. Nagao et al., J. Appl. Crystallogr. 36, 602 (2003).

[2] M. Kotlarchyk et al., Phys. Rev. A 29, 2054 (1984).

[3] H. Seto et al., Phys. Rev. E 54, 629 (1996).

[4] M.W. Kim et al., Phys. Rev. Lett. 54, 46 (1985).

[5] M. Nagao and H. Seto, Phys. Rev. E 59, 3169 (1999).

[6] H. Seto et al., J. Chem. Phys. 112, 10608 (2000).

[7] Y. Kawabata et al., Appl. Phys. A 74 (Suppl.), S534 (2002).

[8] W. Helfrich, Z. Naturforsch. 28C, 693 (1973).

[9] J. S. Huang et al., Phys. Rev. Lett. 59, 2600 (1987).

[10] B. Farago et al., Phys. Rev. Lett. 65, 3348 (1990).

[11] T. Hellweg and D. Langevin, Physica (Amsterdam) 264A, 370 (1999).

[12] Y. Kawabata et al., J. Phys. Soc. Jpn. Suppl. A 70, 445 (2001).

[13] M. Gradzielski et al., J. Phys. Chem. 99, 13232 (1995).

[14] S. T. Milner and S. A. Safran, Phys. Rev. A 36, 4371 (1987).

[15] K. Seki and S. Komura, Physica (Amsterdam) 219A, 253 (1995).

[16] Y. Kawabata et al., J. Neutron Res. 10, 131 (2002).

[17] A. Würger, Phys. Rev. Lett. 85, 337 (2000).

[18] M. Nagao et al., J. Chem. Phys. 115, 10036 (2001).

[19] A. G. Zilman and R. Granek, Phys. Rev. Lett. 77, 4788 (1996). 